\title{
Competition between excitons and exciplexes: Experiments on multilayered organic light emitting diodes
}

\author{
M. Castellani ${ }^{\text {a) }}$ \\ Laboratoire d'Optoélectronique des Matériaux Moléculaires, Ecole Polytechnique Fédérale (EPFL), \\ CH-1015 Lausanne, Switzerland \\ D. Berner \\ CFG Microelectronics S.A., CH-1110 Morges, Switzerland
}

(Received 30 January 2007; accepted 11 June 2007; published online 23 July 2007)

\begin{abstract}
The electronic processes responsible for charge transport and electroluminescence in multilayered organic light emitting diodes (OLEDs) are very sensitive to the properties of the organic heterojunction. In particular, the height of the energy barrier affects the way in which electrons and holes meet at the heterojunction, the way in which the barrier is crossed, and the probability for photon creation. We investigate these aspects experimentally using a family of OLED devices in which different hole transporting materials are used in otherwise identical device architectures to vary the interfacial hole barrier over a wide energy range. We find that the quantum efficiency of the device is maximum for low-energy barriers and drops for high barrier values where a redshifted electroluminescence spectrum is observed. This shift is attributed to exciplex generation at the heterojunction. The contributions of exciton and exciplex annihilation in radiative and nonradiative channels to the charge flow within the heterojunction region are separated and quantified. (C) 2007 American Institute of Physics. [DOI: 10.1063/1.2757204]
\end{abstract}

\section{INTRODUCTION}

Since the early days of organic light emitting diodes (OLEDs), it has been recognized that interfaces play a crucial role in device performance. ${ }^{1,2}$ It is very often the case that charge energy barriers created at interfaces affect charge injection and recombination more than the conducting properties of the organic material itself. Indeed, many efforts to improve OLEDs have been directed toward the optimization of the inorganic-organic interfaces. For example, different treatments of the indium tin oxide (ITO) anode, such as oxygen plasma cleaning, ${ }^{3}$ or grafting of monomolecular layers as interfaces ${ }^{4}$ have been tried.

Another well-established path toward efficient OLEDs is the preparation of organic multilayered structures. In these devices the light is usually generated close to the interface between the electron transporting layer (ETL) and the hole transporting layer (HTL). The energy barrier at this organic/ organic interface affects the probability and the very nature of the recombination process. In most devices, exciton formation and recombination occur within the same material, i.e., the emitting layer (EL). This layer usually coincides with one of the transport layers, e.g., the electron transporting layer. The recombination is particularly pronounced in the region very close to the heterojunction because of the high concentration of carriers. However, the heterojunction promotes another interesting phenomenon: The formation of exciplexes. Such states are formed when an electron and a hole separately localized on neighboring molecules of different chemical nature are bonded as a result of the Coulomb interaction. As already pointed out in the literature, ${ }^{5}$ and re-

${ }^{a)}$ Electronic mail: mauro.castellani@gmx.ch affirmed in this paper, the difference in ionization potentials of the two materials constituting the heterojunction (the ETL and HTL) is the primary factor governing exciplex formation and decay. It is of great interest, therefore, to know the relative contributions of exciton and exciplex states to charge transport near the heterojunction as well as to light production. This picture is detailed further on.

It should be emphasized that exciplex emission has been studied to date only in a relatively small number of works. ${ }^{5-12}$ In these, exciplex emission is often reported to be redshifted with respect to the bulk EL emission. ${ }^{10,12}$ This is related to the energy difference between the highest occupied molecular orbital (HOMO) level (ionization potential) of the hole transporting material and the lowest-unoccupied molecular orbital (LUMO) level (electron affinity) of the electron transporting material, which favors generation of bound states of lower energy rather than excitons. The radiative decay time is also observed to be generally longer for exciplexes than for excitons. ${ }^{5,12}$ In this experiment, exciplex emission was mostly accompanied by exciton emission, resulting in the multiple component spectra, ${ }^{5,6,10,11}$ an effect which has been exploited to produce almost white emitting OLEDs. ${ }^{5,6}$ We will show that in our devices the exciplex emission is voltage dependent, a phenomenon also found in other works ${ }^{11,12}$ wherein the related mechanism of color tuning is discussed. Because the fraction of radiative exciplex recombination ${ }^{7}$ is small, the appearance of exciplexes generally lowers the efficiency of OLEDs. Exciplex related investigations in OLEDs have also been conducted in relation to aging: Giebeler et $\mathrm{al}^{7}{ }^{7}$ considered the effects of heat or electrical stress on exciplex emitting OLEDs and found a reduction of exciplex emission as a function of time. In another paper, ${ }^{8}$ exciplex emission was used to estimate the mobility 

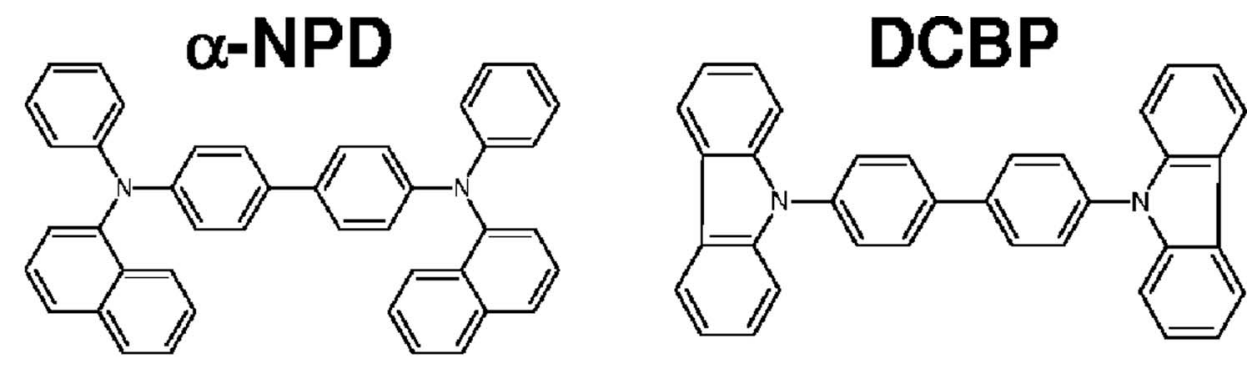

TCTA

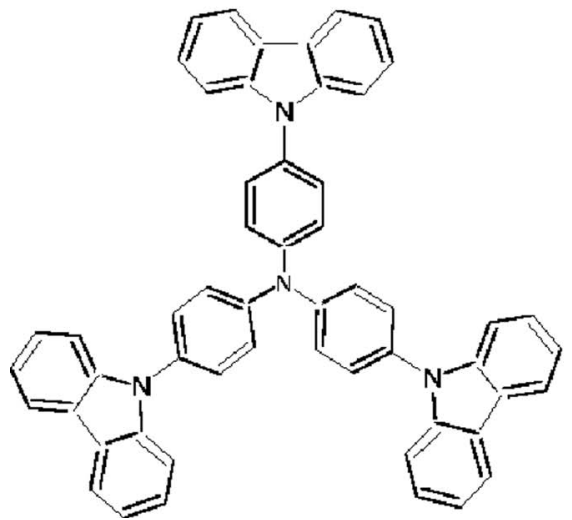

\section{T-NATA}

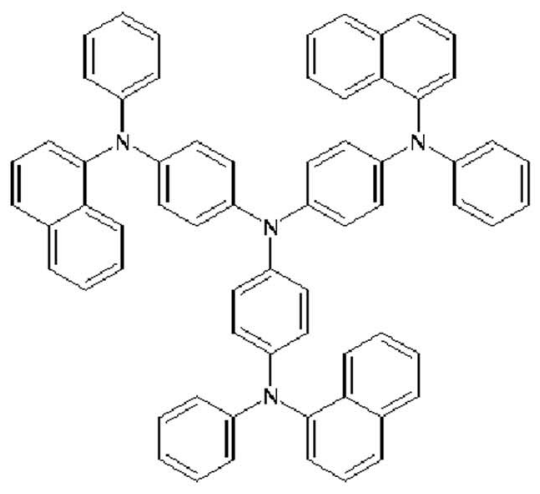

FIG. 1. Molecular structures of hole transporting materials used. $\alpha$-NPD, $N, N^{\prime}$-diphenyl- $N, N^{\prime}$-bis(1-naphthyl)-1, $1^{\prime}$ biphenyl-4, $4^{\prime \prime}$ diamine; TCTA, 4,4', 4"-tris(carbazol-9-yl)-triphenylamine) or DCBP (4,4'-bis(carbazol-9yl)-biphenyl; 1T-NATA, 4,4', 4"tris( $N$-(1-naphtyl)- $N$-phenyl-amino)-triphenylamine; DCBP, 4, 4'-bis(carbazol-9-yl)-biphenyl.

of one of the constituent organic materials of the OLED. Organic heterojunctions and exciplex formation also play extremely important roles in organic photovoltaics. ${ }^{9}$

The present paper focuses on the influence of the energy barrier imposed by the heterojunction on the efficiency of OLEDs. We analyze the rates of exciton and exciplex formation and the relative strengths of their radiative and nonradiative recombination channels. In particular, in this work, we quantify the contribution of exciplexes to nonradiative recombination at a heterojunction in multilayered OLEDs. This paper is organized as follows. The next section contains the experimental details related to the preparation of devices used in this study. Section III contains the results of the experimental characterization of these devices. The data are discussed and analyzed in Sec. IV.

\section{DEVICE FABRICATION AND CHARACTERIZATION}

For this study, a set of OLED devices was prepared employing different HTL materials in order to vary the hole transfer energetics at the HTL/ETL interface. In all cases $\mathrm{Alq}_{3}$ (tris(8-hydroxyquinolato) aluminium) was used as the ETL compound. The general device architecture and the preparation procedure of the multilayer diodes were as follows. The ITO coated glass substrates (18 $\Omega / \square$, Merck) were first cleaned in ethanol, acetone, and soap ultrasonic baths. Prior to evaporation of the organic materials the ITO electrodes were treated by oxygen plasma (0.2 mbar pressure) for $4 \mathrm{~min}$ at $10 \mathrm{~W}$, for work function tuning and chemical cleaning. The variable hole transporting layer, $40 \mathrm{~nm}$ thick, was subsequently evaporated, using one of the four following compounds: $\alpha$-NPD ( $N, N^{\prime}$-diphenyl$N, N^{\prime}$-bis(1-naphthyl)-1, $1^{\prime}$ biphenyl-4, $4^{\prime \prime}$ diamine), TCTA (4, $4^{\prime}, 4^{\prime \prime}$-tris(carbazol-9-yl)-triphenylamine), DCBP $\left(4,4^{\prime}\right.$ bis(carbazol-9yl)-biphenyl), or 1T-NATA $\left(4,4^{\prime}, 4^{\prime \prime} \operatorname{tris}(N-(1-\right.$ naphtyl)- $N$-phenyl-amino)-triphenylamine). The molecular structures of these compounds are shown in Fig. 1. In some devices, a second hole-transport layer based on TCTA was introduced with either 2 or $10 \mathrm{~nm}$ thickness. To separate the recombination zone from the cathode, a $60 \mathrm{~nm}$ thick electron transport layer of $\mathrm{Alq}_{3}$ was deposited. This acted simultaneously as an emitting material. All organics, previously purified by gradient sublimation, were thermally evaporated at a rate of $1.0 \AA / \mathrm{s}$ at a base pressure of around 5 $\times 10^{-7}$ mbar. A $0.8 \mathrm{~nm} \mathrm{LiF} \mathrm{layer} \mathrm{was} \mathrm{deposited} \mathrm{right} \mathrm{after}$ the $\mathrm{Alq}_{3}(0.2 \AA / \mathrm{s})$. The finishing $\mathrm{Al}$ electrode (cathode) was deposited $(10 \AA / s)$ in another chamber without breaking the vacuum. This complex device structure is depicted by means of an energy diagram in Fig. 2. The active area of the diode segments was $10 \mathrm{~mm}^{2}$.

The current-versus-voltage charactestics of each device were measured in a nitrogen atmosphere glove box by means of a LABVIEW controlled Keithley 236 measuring unit. Simultaneously, the light output was measured by a photodiode, calibrated with a Minolta LS110 luminance meter. Electroluminescence spectra were recorded by an Ocean Optics Inc. S2000 fiber optic spectrometer at different currents (0.5 and $5 \mathrm{~mA}$ ). For the calculation of the quantum efficiency Lambert emission was taken into account. The HOMO levels 


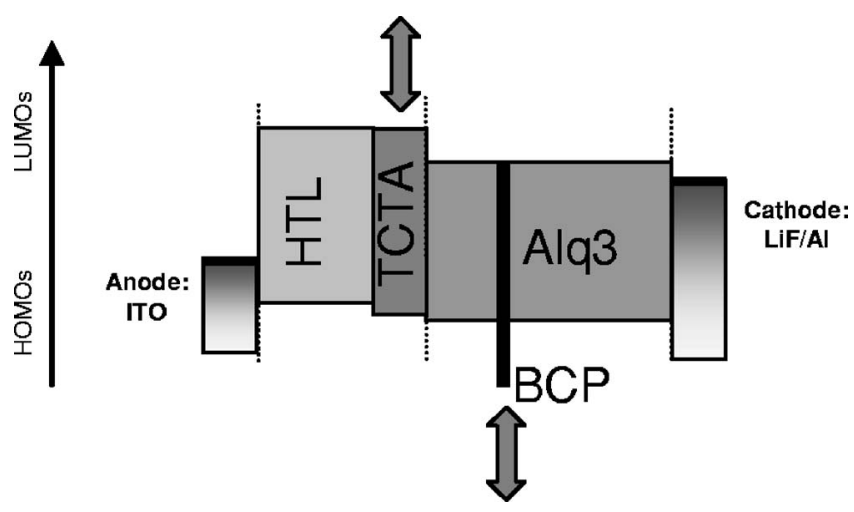

FIG. 2. Schematic energy level representation of the OLEDs fabricated. Bathocuproine (BCP) is used as hole-blocking layer in a separate experiment.

were measured in our laboratory by using the cyclic voltametry method in solution. Table I summarizes the six different types of the devices fabricated and introduces the respective labels to be used later in the text.

\section{RESULTS}

The most important parameters characterizing the interface between the hole transporting and the electron transporting layer in our devices are the HOMO levels of the two materials. This is because the heterojunction energy barriers for the electrons (i.e., the differences of LUMO levels) are always so high (on the order of $0.7 \mathrm{eV}$ or bigger) that the process of electron crossing without the involvement of holes may be regarded as negligible. The values of the HOMO levels obtained by cyclic voltametry measurements are given in Fig. 3 and are in good agreement with those found in the literature. In most cases, the difference between the HOMO level of the hole-transport material and the HOMO level of $\mathrm{Alq}_{3}$ acts as an energetic barrier that impedes the injection of holes from the HTL into the ETL $\left(\mathrm{Alq}_{3}\right)$. These differences are given on the right hand side of Fig. 3. The highest barrier in our devices was found in the 1T-NATA device. Much lower barriers for holes were found in devices containing TCTA or $\alpha$-NPD. There is no apparent barrier at the heterojunction when DCBP is used as a hole transporter (zero-order approximation).

We first present the results for devices which include either $\alpha$-NPD (device A), TCTA (device D), or both (devices $\mathrm{B}$ and $\mathrm{C}$ ). The current-versus-voltage curves as well as the dependence of the external quantum efficiency on current are given in Figs. 4(a) and 4(b), respectively. The principal conclusion from these graphs is that devices which contain TCTA near the heterojunction are more efficient than those

TABLE I. Device architectures used in this study (the full names of the chemical compounds are specified in the text).

\begin{tabular}{llll}
\hline \hline (A) & ITO $\left(\right.$ plasma $\left.\mathrm{O}_{2}\right) / \alpha$-NPD & & /Alq3/LiF-Al \\
$(\mathrm{B}, \mathrm{C})$ & ITO $\left(\right.$ plasma $\left.\mathrm{O}_{2}\right) / \alpha$-NPD/ & TCTA $(2,10 \mathrm{~nm})$ & /Alq3/LiF-Al \\
$(\mathrm{D})$ & ITO $\left(\right.$ plasma $\left.\mathrm{O}_{2}\right) /$ & TCTA $(40 \mathrm{~nm})$ & /Alq3/LiF-Al \\
(E) & ITO $\left(\right.$ plasma $\left.\mathrm{O}_{2}\right) /$ & 1T-NATA $(40 \mathrm{~nm})$ & /Alq3/LiF-Al \\
(F) & ITO $\left(\right.$ plasma $\left.\mathrm{O}_{2}\right) /$ & DCBP $(40 \mathrm{~nm})$ & /Alq3/LiF-Al \\
\hline \hline
\end{tabular}

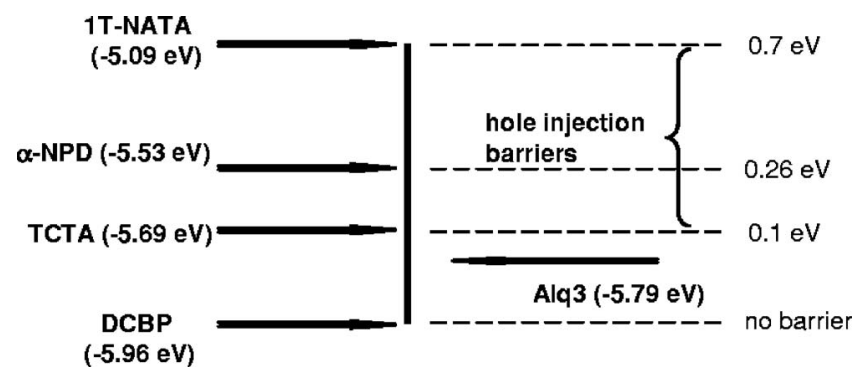

FIG. 3. Energy level scheme at different (ETL/HTL) heterojunctions, with hole barriers given with respect to $\mathrm{Alq}_{3}$ (zero-order approximation).

which contain only $\alpha$-NPD. The thickness of the TCTA layer turns out not to be particularly important in this respect. The $2 \mathrm{~nm}$ thick TCTA OLED is almost as good as the one with $10 \mathrm{~nm}$, and these devices do not differ very much from the one where the full $40 \mathrm{~nm}$ of HTL is composed of TCTA only. Hence, the efficiency is primarily related to the composition of the "bipolar" heterojunction, where electrons and holes meet and where most of the recombination takes place. It is less related to the details of the device structure far from that heterojunction. It may be also observed that the $I(V)$ curves for devices A-D are very similar. This is easily understood from the fact that the current-voltage curves are primarily
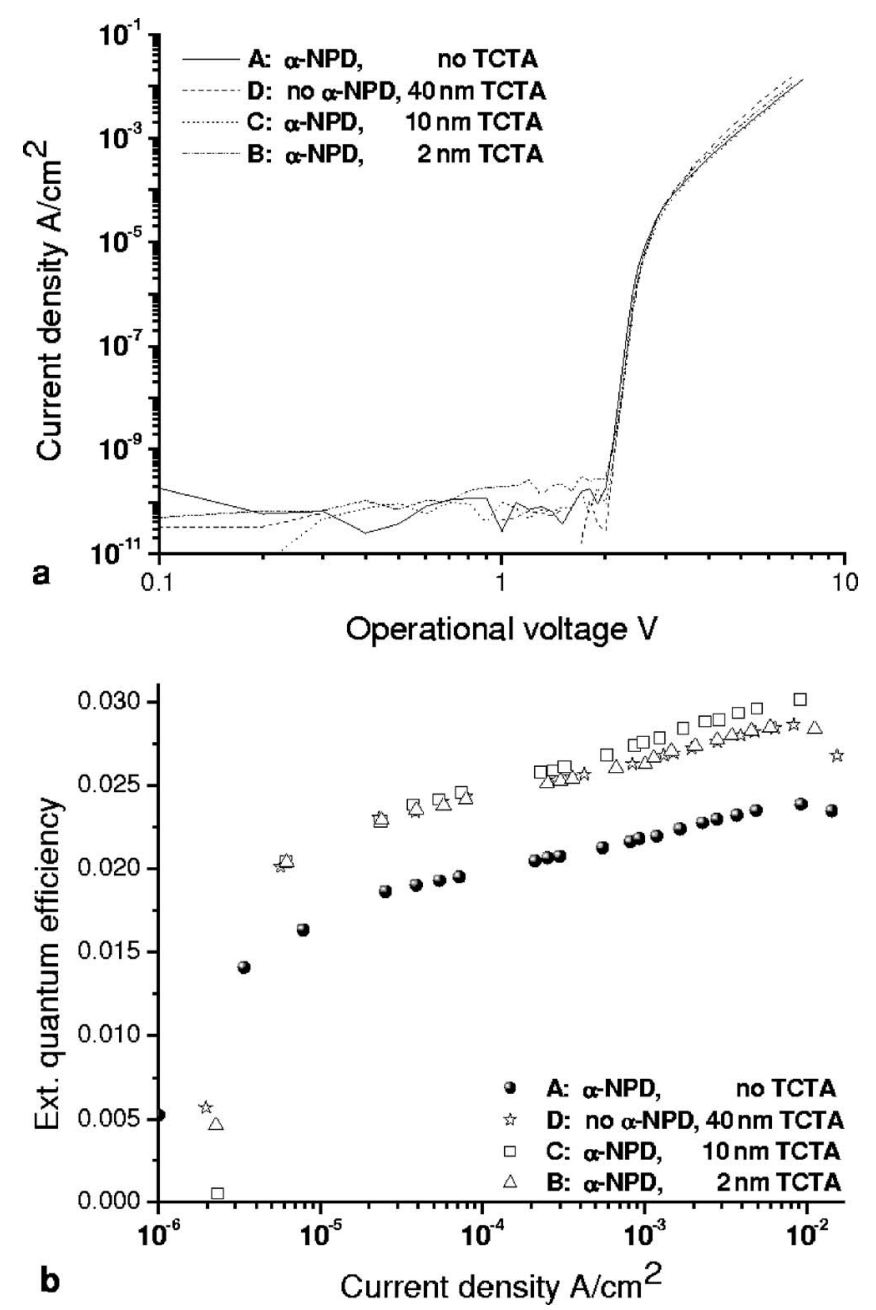

FIG. 4. Current density vs voltage $[J(V)]$ characteristics (a) and external quantum efficiency as a function of current density (b) for devices A-D. 
TABLE II. OLED performance parameters for devices A-D. The "injection voltage" marks the transition from the injection limited current regime to the space charge limited current regime; the "injection slope" corresponds to the derivative of $I(V)$ at the transition. The external quantum efficiencies and energetic efficiencies are given at a luminance of $100 \mathrm{~cd} / \mathrm{m}^{2}$.

\begin{tabular}{lccccc}
\hline \hline $\begin{array}{c}\text { HTL layer } \\
\text { structure }\end{array}$ & $\begin{array}{c}\text { Injection } \\
\text { slope [- }\end{array}$ & $\begin{array}{c}\text { Injection } \\
\text { voltage }(\mathrm{V})\end{array}$ & $\begin{array}{c}\text { Voltage for } \\
100 \mathrm{~cd} / \mathrm{m}^{2}\end{array}$ & $\begin{array}{c}\text { Ext. eff. at } \\
100 \mathrm{~cd} / \mathrm{m}^{2} \\
(\%)\end{array}$ & $\begin{array}{c}\text { En. eff at } \\
100 \mathrm{~cd} / \mathrm{m}^{2} \\
(\mathrm{~lm} / \mathrm{W})\end{array}$ \\
\hline$\alpha$-NPD only & 53.23 & 1.9 & 3.3 & 2.02 & 6.13 \\
Int. TCTA 10 nm & 51.85 & 2.0 & 3.4 & 2.42 & 7.57 \\
Int TCTA 2 nm & 50.43 & 2.0 & 3.3 & 2.45 & 7.95 \\
TCTA only & 47.27 & 2.0 & 3.3 & 2.48 & 8.06 \\
\hline \hline
\end{tabular}

dictated by injection at the electrodes and space charge effects (mostly inside $\mathrm{Alq}_{3}$ ) and not by the heterojunction. This has been discussed in many papers related to bilayer molecular OLEDs, and modeled in detail for a structure similar to ours in Ref. 13. In particular, the robustness of the $I(V)$ characteristics in devices A-D may be related to the stability of the injection barrier value even upon change of the hole transport layer. ${ }^{14,15}$

The key performance parameters measured for the four devices are listed in Table II. The injection slope listed in Table II does not vary considerably between devices A, B, C, and $\mathrm{D}$. These devices behave as virtually equal also at higher voltages with light output above $100 \mathrm{~cd} / \mathrm{m}^{2}$, where considerable space charge effects within $\mathrm{Alq}_{3}$ are expected. The only signficant difference is the $25 \%$ increase in the external quantum and energetic efficiencies of devices B, C, and D, i.e., those with TCTA as the HTL at the bipolar heterojunction, over OLED A which has only $\alpha$-NPD as the hole transport layer.

In comparison with devices A-D, the heterojunctions in devices $\mathrm{E}$ and $\mathrm{F}$ are very different. The performances of these devices using 1T-DATA and DCBP as the HTL, respectively, are shown in Fig. 5 and Table III. Device E is characterized by a very high-energy barrier for holes at the heterojunction, its bare value being approximately $0.7 \mathrm{eV}$ (see Fig. 3). In contrast, no apparent barrier for holes seems to

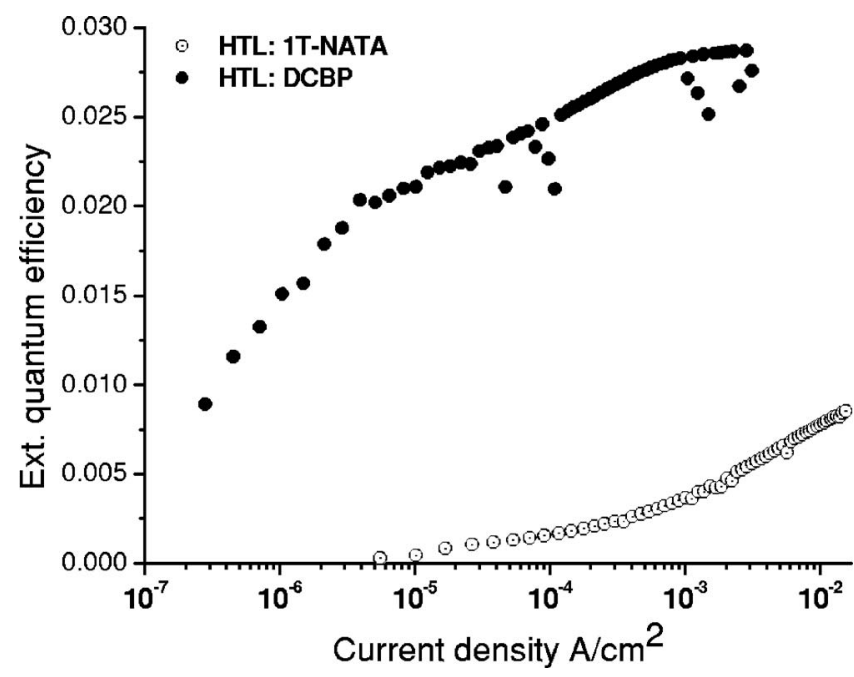

FIG. 5. Quantum efficiencies as a function of operating current density for devices $\mathrm{E}$ (high hole barrier at heterojunction) and $\mathrm{F}$ (no hole barrier at heterojunction). exist in device $\mathrm{F}$. The efficiency of device $\mathrm{E}$ is very much lower (some five times at $100 \mathrm{~cd} / \mathrm{m}^{2}$ ) than the efficiency of device $\mathrm{F}$, which is comparable to those of devices A-D, although slightly less efficient than, those based on TCTA. Even more interesting is the comparison of the electroluminescent spectra. Normalized spectra of all devices are shown in Figs. 6 and 7 for two values of the operating current. The characteristic parameters are shown into Tables IV and V. At both values of the current, 0.5 and $5 \mathrm{~mA}$, there is an observable shift of the spectrum of device $\mathrm{E}$ with respect to the spectra of the other devices. This is seen in both Figs. 6 and 7 and in the parameters $\lambda_{\max }$ and full width at half maximum (FWHM) in Tables IV and V. The shift, more pronounced for low than for high operating current, is visible only for devices with a very high barrier at the heterojunction. These findings will be analyzed in detail in the next section.

\section{DISCUSSION}

The change of material in the hole transport layer simultaneously affects the organic heterojunction and the anodeorganic contact. In order to eliminate the effect of the latter, the quantum efficiency of the devices as a function of the total current density is compared. Devices B-D show that the efficiency versus current is not significantly affected by device structure far from the bipolar heterojunction. In contrast, we observe that the efficiency depends on the height of the energetic barrier at the bipolar heterojunction. The efficiency of high barrier devices (E) based on 1T-NATA is very low, while the efficiency is much higher in $\alpha$-NPD based devices (A), where the barrier is reduced by $0.44 \mathrm{eV}$. Further reduction of the barrier by $0.16 \mathrm{eV}$, accomplished by the replacing $\alpha$-NPD with TCTA (devices B-D), increases the quantum gain further by approximately $25 \%$. The efficiency is at its highest with the TCTA/ $/ \mathrm{Alq}_{3}$ heterojunction. A further move to DCBP-based devices where no barrier exists begins to

TABLE III. Luminance and (external quantum and energetic) efficiencies for devices $\mathrm{E}$ (high hole barrier at heterojunction) and $\mathrm{F}$ (no nominal hole barrier at heterojunction).

\begin{tabular}{lccc}
\hline \hline $\begin{array}{c}\text { HTL layer } \\
\text { material }\end{array}$ & $\begin{array}{c}\text { Voltage for } \\
100 \mathrm{~cd} / \mathrm{m}^{2}\end{array}$ & $\begin{array}{c}\text { Ext. eff. at } \\
100 \mathrm{~cd} / \mathrm{m}^{2} \\
(\%)\end{array}$ & $\begin{array}{c}\text { En. eff at } \\
100 \mathrm{~cd} / \mathrm{m}^{2} \\
(\mathrm{~lm} / \mathrm{W})\end{array}$ \\
\hline 1T-NATA & 4.5 & 0.40 & 0.92 \\
DCBP & 5.0 & 2.06 & 5.15 \\
\hline \hline
\end{tabular}




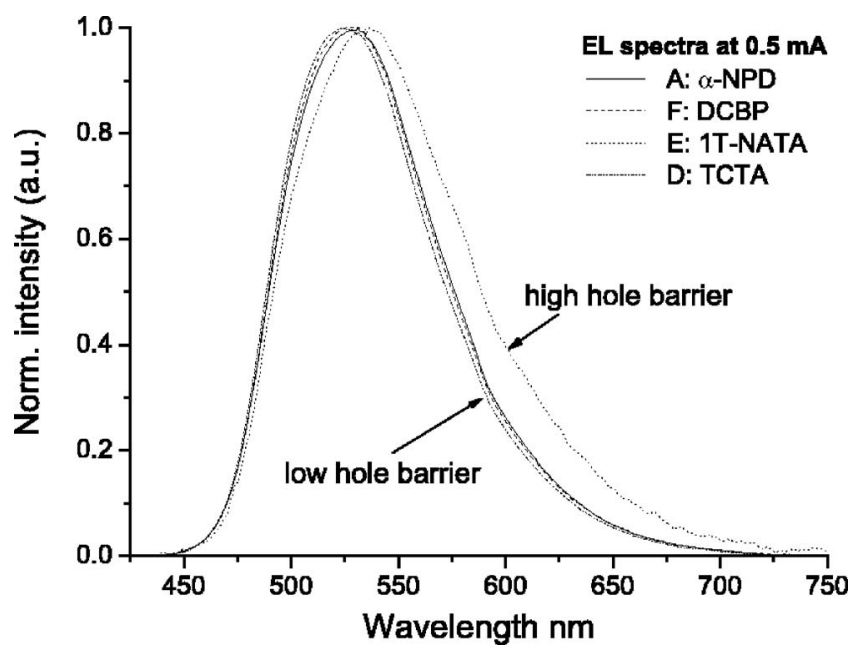

FIG. 6. Electroluminescent spectra of devices with different HTL materials at $0.5 \mathrm{~mA}$ : A ( $\alpha$-NPD), F (DCPB), E (1T-NATA), and D (TCTA).

reduce the efficiency, at least at low-to-moderate currents. It should be remarked that this reduction is rather small, possibly related to the extremely low mobility of holes in $\mathrm{Alq}_{3}$. As illustrated in Ref. 13, this low mobility hampers holes from reaching the cathode before being recombined. Experimentally, this was checked using a separate device wherein a thin hole-blocking layer of electron-transport material bathocuproine (BCP) was inserted into the $\mathrm{Alq}_{3}$ layer, as shown in Fig. 2. The result (not shown) was a rise of the quantum efficiency, but negligible in comparison with the change in efficiency accomplished by manipulating the barrier height at hetereojunction. Therefore, the percentage of holes that leak toward the electrode can be regarded as negligible.

The redshift of the spectra seen in the low-efficiency 1T-NATA-based devices gives an indication of the source of the efficiency variation when barrier height is changed. We propose that the shift is due to the recombination of an electron-hole pair building an excited state extended on two neighboring molecules of different chemical nature. We will call it exciplex in the following, although in the literature also other terms such as charge transfer complex are used for

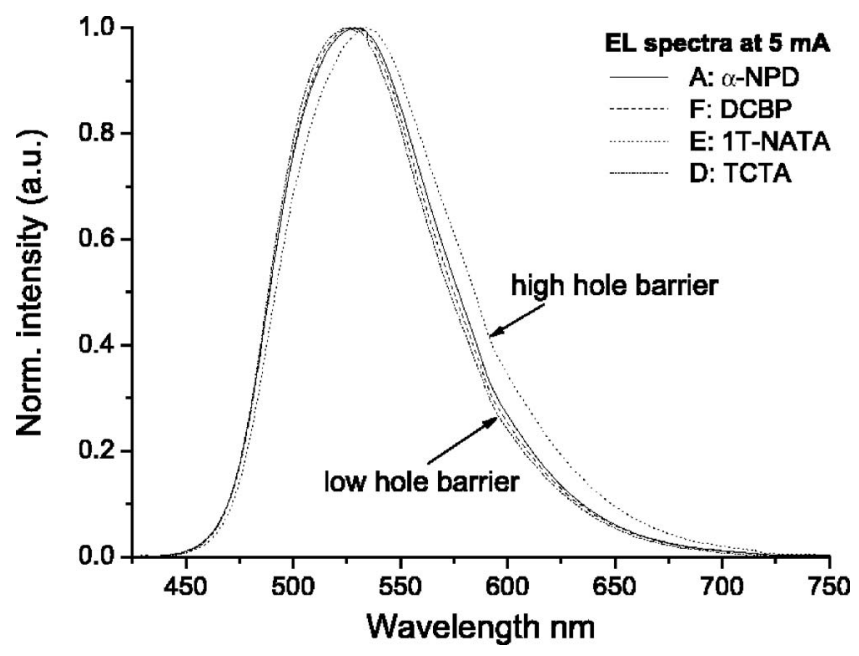

FIG. 7. Electroluminescent spectra of devices with different HTL materials at $5 \mathrm{~mA}$ : A ( $\alpha$-NPD), F (DCPB), E (1T-NATA), and D (TCTA).
TABLE IV. Full width at half maximum (FWHM) and wavelength of maximum emission intensity $\left(\lambda_{\max }\right)$ of electroluminescent spectra for devices A, $\mathrm{D}, \mathrm{E}$, and $\mathrm{F}$ at $0.5 \mathrm{~mA}$.

\begin{tabular}{ccc}
\hline HTL & $\lambda_{\max }(\mathrm{nm})$ & FWHM $(\mathrm{nm})$ \\
\hline 1T-NATA & 534.61 & 96.98 \\
$\alpha$-NPD & 529.05 & 86.79 \\
TCTA & 525.58 & 84.08 \\
DCBP & 526.97 & 86.11 \\
\hline \hline
\end{tabular}

particles of this kind. The energetics of exciplex versus exciton at the heterojunction is schematically shown in Fig. 8. The redshift is an essential feature of exciplex radiation since the electron-hole pair bound in an exciplex is at a lower energy than a pair bound in an exciton. Within the simplified picture of Fig. 8 and using the notation introduced there, the energy difference between the exciplex and exciton states can be written as

$\left(\Delta_{\mathrm{ETL}}-E_{b}^{t}\right)-\left(\Delta_{\mathrm{EX}}-E_{b}^{p}\right)=\Delta_{h}-\left(E_{b}^{t}-E_{b}^{p}\right) \equiv \Delta_{h}-\delta E_{b}$.

The difference in binding energies between excitons and exciplexes, $\delta E_{b}=E_{b}^{t}-E_{b}^{p}$, is expected to be positive, mainly because the Coulomb attraction is stronger for on-site than for off-site configurations. Hence, that the barrier energy $\Delta_{h}$ be high enough, i.e., $\Delta_{h}>\delta E_{b}$, is the main requirement for exciplex stabilization. In the opposite case, the exciplex-toexciton transformation is exothermal. We demonstrate experimentally that at higher operational voltages the exciplex contribution is less important in high barrier devices (compare Figs. 6 and 7): An external voltage drop reduces the hole injection barrier at heterojunction and this in turn reduces the probability of exciplex appearance. The calculations presented below are valid at a specific driving current (high current regime).

Using the idea that the measured spectra of high barrier devices consist of exciton and exciplex components we may analyze those presented in Figs. 6 and 7 to further our understanding of the processes at the heterojunction. The relative energy shift of exciplex and exciton components suggests that even when they leave mixed from the device, the high-energy branch of the electroluminescent spectrum is dominated by excitons. With this in mind, it is rather straightforward to separate the contributions of excitons and exciplexes to the electroluminescent spectrum from our 1T-NATA-based devices. We start with the pure electroluminescent spectrum of $\mathrm{Alq}_{3}$ of devices with low barrier (TCTA based) or no barrier at all (DCBP based), where no exciplex contribution is expected. This spectrum is then rescaled to fit

TABLE V. Full width at half maximum (FWHM) and wavelength of maximum emission intensity $\left(\lambda_{\max }\right)$ of electroluminescent spectra for devices A, $\mathrm{D}, \mathrm{E}$, and $\mathrm{F}$ at $5 \mathrm{~mA}$.

\begin{tabular}{ccc}
\hline \hline HTL & $\lambda_{\max }(\mathrm{nm})$ & FWHM $(\mathrm{nm})$ \\
\hline 1T-NATA & 533.92 & 92.89 \\
$\alpha$-NPD & 529.05 & 88.52 \\
TCTA & 526.27 & 84.42 \\
DCBP & 527.66 & 86.12 \\
\hline \hline
\end{tabular}




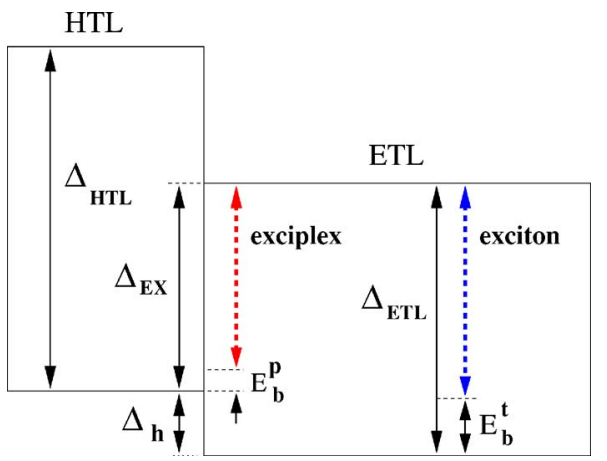

FIG. 8. Energy scheme of electron-hole states formed in bilayer devices. $\Delta_{h}$ is the hole injection barrier at heterojunction. The electronic energy gap of the hole transport material $\left(\Delta_{\mathrm{HTL}}\right)$ is symbolized by arrows. In addition, the "heterojunction energy gap" $\left(\Delta_{\mathrm{EX}}\right)$ is indicated. The two dashed lines represent the effective quasiparticle energies: The exciplex energy is only slightly reduced compared to the heterojunction gap $\left(\Delta_{\mathrm{EX}}\right)$, while the exciton energy is clearly smaller than the ETL energy gap $\left(\Delta_{\mathrm{ETL}}\right)$. The respective differences are the binding energies of exciplex $\left(E_{b}^{p}\right)$ and exciton $\left(E_{b}^{t}\right)$, the latter presumably being bigger, $\delta E_{b} \equiv E_{b}^{t}-E_{b}^{p}>0$.

the high-energy tail of the 1T-NATA-based spectrum. The difference to the high barrier device spectrum is then attributed to exciplex emission. The procedure is illustrated in Fig. 9. Most importantly we can extract from the areas under the supspectra in Fig. 9 the relative emissive contributions of exciplexes, $19 \pm 5 \%$, and excitons, $81 \pm 5 \%$, respectively.

The analysis may be further advanced to identify the contributions from various recombination channels to charge transport. For this, we assume that the leakage of holes toward the cathode may be neglected in the high current regime. The experiment with the thin BCP-based, hole blocking sublayer inserted into $\mathrm{Alq}_{3}$ layer already supports the assumption that the percentage of such holes is small. Another support comes from our detailed experimental study and modeling of related devices. ${ }^{13,16}$ There we have shown that the space charge barrier in $\mathrm{Alq}_{3}$ becomes dominant for higher current and that hole leakage becomes completely negligible. The original study was based on $\alpha$-NPD; it is expected that the hole leakage current is even lower for devices with higher barrier at the heterojunction, i.e., those where 1T-NATA is used for a HTL.

With no electrons entering the HTL and no holes reaching the cathode, each electron crossing the device must invariably be associated with an electron-hole recombination of some sort, with the recombination processes taking place at the heterojunction itself or within the $\mathrm{Alq}_{3}$ layer, not too far from the heterojunction. Four channels of recombination are envisaged to contribute to the total current through the device; any other possibilities are neglected here.

(1) Rt, radiative annihilation of excitons on $\mathrm{Alq}_{3}$ molecules;

(2) $\mathrm{Nt}$, nonradiative annihilation of excitons on $\mathrm{Alq}_{3}$ molecules;

(3) Rp, radiative annihilation of exciplexes at the heterojunction;

(4) $\mathrm{Np}$, nonradiative annihilation of exciplexes at the heterojunction;

where the labels are $\mathrm{R}$ =radiative, $\mathrm{N}=$ nonradiative, $\mathrm{t}$ $=$ exciton, and $\mathrm{p}=$ exciplex. It is also very important to note

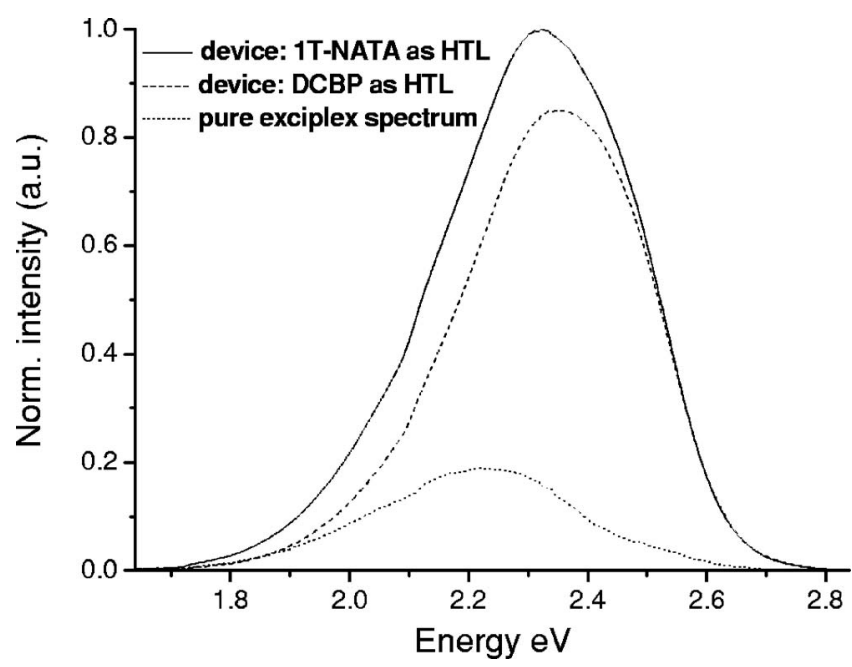

FIG. 9. Normalized energy dispersive electroluminescent spectra of an exciplex emitting device (solid line, E; high hole energy barrier at heterojunction), of an exciton only device (dashed line, F; no hole barrier at heterojunction), and of pure exciplexes (dotted, subtraction of spectra of $\mathrm{E}$ and F), respectively. The deconvolution is explained in the text.

what is meant by "radiative recombination process" here, that is, only those processes which produce photons that actually leave the device and contribute to the external quantum efficiency. Recombination processes producing photons that are subsequently absorbed within the device are counted as nonradiative. This definition turns out to be convenient in the following analysis. (The internal quantum efficiency may be calculated from the external efficiency, once the outcoupling coefficient, i.e., the probability that the photon created in the organic layer finds its way out of the device, is known. ${ }^{17}$ ) Therefore, the total current $I$ is the sum of the contributions $I_{\mathrm{Rt}}, I_{\mathrm{Nt}}, I_{\mathrm{Rp}}$, and $I_{\mathrm{Np}}$ of the four channels,

$$
I=I_{\mathrm{Rt}}+I_{\mathrm{Nt}}+I_{\mathrm{Rp}}+I_{\mathrm{Np}} \text {. }
$$

To determine the four current contributions, three additional conditions are required, which are discussed in the following.

The radiative part, $I_{\mathrm{Rt}}+I_{\mathrm{Rp}}$, is accessible by the experiments and is directly related to measured external quantum efficiency of a high barrier device at a given current,

$$
\eta_{\mathrm{ext}}=\frac{I_{\mathrm{Rt}}+I_{\mathrm{Rp}}}{I} \text {. }
$$

Equation (3) represents the second equation for unknowns $I_{\mathrm{R}}, I_{\mathrm{N}}, I_{\mathrm{Rp}}$, and $I_{\mathrm{Np}}$ in the 1T-NATA device. The third equation, Eq. (4), comes from the analysis of the spectrum in Fig. 9. It sets the ratio of radiative contributions of excitons and exciplexes. It is given by the ratio of integrals of two spectral components; e.g., for $I=5 \mathrm{~mA}$, one obtains

$$
I_{\mathrm{Rt}} / I_{\mathrm{Rp}} \approx 81 / 19 \text {. }
$$

The fourth equation is for the ratio $I_{\mathrm{Rt}} / I_{\mathrm{Nt}}$ of radiative and nonradiative channels of the $\mathrm{Alq}_{3}$ excitonic recombination. In our high barrier device, E, we can presumably introduce for the two types of recombination, either exciplexes or excitons, two different emissive external quantum efficiencies. Since the external quantum efficiency of a high barrier de- 
vice $\left(\eta_{\text {ext }}\right)$ is composed of both contributions, we search among our device family for samples that exhibit pure emission of one exited state type, e.g., excitons. We deduce in the following procedure the corresponding emissive efficiency term, $\eta_{\mathrm{Alq}_{3}}$, for the bulk singlet excitons deriving from the EL.

Figures 4 and 5 suggest that the family of devices can be divided into three groups by using the external quantum efficiency as a criterion (at $5 \mathrm{~mA}$, high current regime).

(i) No and low barrier devices (samples F and D, respectively): The quantum efficiency is around $\sim 2.9 \%$ for both types of devices and their derivatives (samples $\mathrm{B}$ and $\mathrm{C}$ ).

(ii) Intermediate barrier device (sample A): The quantum efficiency is slightly reduced to $\sim 2.3 \%$.

(iii) High barrier device (sample E): The quantum efficiency drops to $\sim 0.6 \%$.

Since the first device type (i) includes devices exhibiting different hole barriers at heterojunction, but about the same maximum efficiency, we conclude that exciplex emission is negligible therein. Consequently, the quantum efficiency of these devices corresponds to the bulk singlet quantum efficiencies of the $\mathrm{Alq}_{3}$ emissive layer as defined in Eq. (5):

$$
\eta_{\mathrm{Alq}_{3}} \approx \frac{I_{\mathrm{Rt}}}{I_{\mathrm{Rt}}+I_{\mathrm{Nt}}} .
$$

Note that Eq. (5) has been derived for a pure exciton emitting low barrier device (device type i); therefore, exciplex related recombination channels can be omitted and the total current is given by $I_{\mathrm{Rt}}$ and $I_{\mathrm{Nt}}$ only. Or by other means, the values $I_{\mathrm{Rt}}$ and $I_{\mathrm{Nt}}$ strongly depend on the device type. They are both higher in low barrier devices than in high barrier devices, since in the latter the total current is given by four addends [see Eq. (2)]. On the other hand, the experimental value $\eta_{\mathrm{Alq}_{3}}$ is an intrinsic property of the EL and is therefore independent of the heterojunction properties. This is correct when the outcoupling coefficient for the photons coming from exciton annihilation does not change significantly among the different devices. Since the EL is made of the very same material and thickness in all of our devices we will generalize Eq. (5) to all of them, including the high barrier diodes. This important step is justified in the following.

As pointed out earlier in this paper, transport of holes inside the EL is governed by the space-charge mobility. ${ }^{13,16}$ Therefore, the recombination rate of holes is determined only by this bulk property and not affected by the heterojunction characteristics. Consequently, the $I_{\mathrm{Rt}} / I_{\mathrm{Nt}}$ ratio can be considered constant for all devices of this study. From Eq. (5), this ratio can be isolated, leading to the equivalent Eq. (6), which obviously only depends on the emissive quantum efficiency of the recombination zone:

$$
\frac{I_{\mathrm{Rt}}}{I_{\mathrm{Nt}}} \approx \frac{\eta_{\mathrm{Alq}_{3}}}{1-\eta_{\mathrm{Alq}_{3}}} .
$$

Altogether this provides four linear equations: Eqs. (2)-(4) and (6), from which unknowns $I_{\mathrm{Rt}}, I_{\mathrm{Nt}}, I_{\mathrm{Rp}}$, and $I_{\mathrm{Np}}$ of the

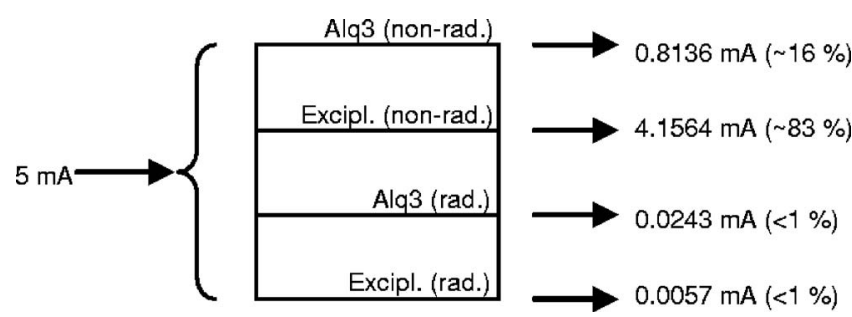

FIG. 10. Decomposition of the total current ( $5 \mathrm{~mA}$ case) in the 1T-NATAbased device into contributions from the four recombination channels. Nt, nonradiative exciton recombination, $\mathrm{Np}$, nonradiative exciplex recombination; Rt, radiative exciton recombination; $\mathrm{Rp}$, radiative exciplex recombination.

1T-NATA device are readily calculated. This decomposition is illustrated in Fig. 10 at an operational current of $5 \mathrm{~mA}$. The component $I_{\mathrm{Np}}$ due to nonradiative exciplex recombination turns out to be the dominant one, and the external quantum efficiency of exciplexes is as low as $0.14 \%$. The latter value can be obtained by applying the definition of the emissive quantum efficiency of exciplexes $\left(\eta_{p}\right)$ to Fig. 10:

$$
\eta_{\mathrm{p}} \approx \frac{I_{\mathrm{Rp}}}{I_{\mathrm{Np}}+I_{\mathrm{Rp}}} .
$$

Thus, the low efficiency of the 1T-NATA device is the consequence of a heterojunction that favors exciplex formation and direct recombination at this organic/organic interface. This picture is summarized in the next section.

\section{CONCLUSIONS}

We have investigated experimentally the influence of energy barrier height at bipolar organic heterojunctions in OLED devices on charge transport and photon generation. The efficiency is found to depend on the barrier height and to have a maximum for low barrier values. The electroluminescent spectra of devices with high-energy barriers show an extra contribution that is redshifted with respect to the spectrum of the emitter material. This extra contribution is voltage dependent and is identified as exciplex emission. We have quantified the contribution of the four major recombination processes to the charge transport and have obtained the absolute contributions of radiative and nonradiative annihilations of excitons and exciplexes to the total current in high barrier devices.

If the barrier at the heterojunction is low, it is responsible for a rather sharp rise of the efficiency at low current values, and it promotes accumulation of holes and electrons on opposite sides of the heterojunction. This in turn enhances the probability of recombination even at intermediate driving voltages. A photon is likely to result for low-to-moderate barrier values since the exciplex state is then unstable toward $\mathrm{Alq}_{3}$ exciton formation. The instability vanishes for higher energy barriers since the difference between the binding energies of the exciton and the exciplex may not compensate for the difference in HOMO levels. Direct recombination of exciplexes at the heterojunction then becomes the dominant decay channel, wherein the electron hops from the LUMO level of one molecule type to the empty HOMO level (i.e., hole) of the other molecule type. However, the fraction of 
radiative annihilations in this case is much smaller than for $\mathrm{Alq}_{3}$ excitons. This is the reason behind the efficiency drop with increasing barrier height. Exciplex annihilation dominantly contributes to the current through the heterojunction while exciton annihilation still dominates the photon production.

It is hoped that the work presented here will stimulate further investigations in several directions. On the experimental side, similar work on structured heterojunctions where Coulomb effects and bound state formation tend to be more involved would be of great interest. ${ }^{18}$ On the theoretical side, other simulations could consider hole crossing at the heterojunction with assistance of electrons, namely, exciplexexciton tansformations.

\section{ACKNOWLEDGMENTS}

The authors would like to express their gratitude to $\mathrm{M}$. Schaer, Ph. Bugnon, and M. Longchamp for their technical support. E. Tutiš and L. Zuppiroli are acknowledged for scientific discussions. One of the authors (M.C.) acknowledges the ETH-Board (council of the Swiss Federal Institutes of Technology) for the research scholarship grant. F. Nüesch, W. Leo and F. N. Nunalee are thanked for critical reading of the manuscript.

${ }^{1}$ C. W. Tang and S. A. Van Slyke, Appl. Phys. Lett. 51, 913 (1987).

${ }^{2}$ S. A. Van Slyke, C. H. Chen, and C. W. Tang, Appl. Phys. Lett. 69, 2160
(1996).

${ }^{3}$ J. S. Kim, M. Granstrom, R. H. Friend, N. Johansson, W. R. Salaneck, R. Daik, W. J. Feast, and F. Cacialli, J. Appl. Phys. 84, 6859 (1998).

${ }^{4}$ L. Zuppiroli, L. Si-Ahmed, K. Kamaras, F. Nuesch, M. N. Bussac, D. Ades, E. Moons, and M. Gratzel, Eur. Phys. J. B 11, 505 (1999).

${ }^{5}$ J. Feng, F. Li, W. B. Gao, S. Y. Liu, Y. Liu, and Y. Wang, Appl. Phys. Lett. 78, 3947 (2001)

${ }^{6}$ M. Berggren, G. Gustafsson, O. Inganäs, M. R. Andersson, T. Hjertberg, and O. Wennerström, J. Appl. Phys. 76, 7530 (1994).

${ }^{7}$ C. Giebeler, H. Antoniadis, D. D. C. Bradley, and Y. Shirota, J. Appl. Phys. 85, 608 (1999).

${ }^{8}$ Y. Kawabe and J. Abe, Appl. Phys. Lett. 81, 493 (2002).

${ }^{9}$ J. J. M. Halls, J. Cornil, D. A. dos Santos, R. Silbey, D. H. Hwang, A. B. Holmes, J. L. Bredas, and R. H. Friend, Phys. Rev. B 60, 5721 (1999).

${ }^{10}$ M. Cocchi, D. Virgili, G. Giro, V. Fattori, P. Di Marco, J. Kalinowski, and Y. Shirota, Appl. Phys. Lett. 80, 2401 (2002).

${ }^{11}$ K. Itano, H. Ogawa, and Y. Shirota, Appl. Phys. Lett. 72, 636 (1998).

${ }^{12}$ L. M. Herz, R. H. Friend, and C. Silva, Phys. Rev. Lett. 92, 247402 (2004).

${ }^{13}$ E. Tutiš, D. Berner, and L. Zuppiroli, J. Appl. Phys. 93, 4594 (2003).

${ }^{14}$ E. Tutiš, M. N. Bussac, and L. Zuppiroli, Appl. Phys. Lett. 75, 3880 (1999).

${ }^{15}$ I. H. Campbell and D. L. Smith, Appl. Phys. Lett. 74, 561 (1999).

${ }^{16}$ D. Berner, H. Houili, W. Leo, and L. Zuppiroli, Phys. Status Solidi A 202, 9 (2005).

${ }^{17}$ The outcoupling coefficient is often estimated to be $20 \%$, based on the probability that the photon reaching the external surface of the device will suffer total reflection. However, the precise value may deviate from this value, depending on the precise geometry of the device, the surface topography, the position of the emitting molecules, as well as on electromagnetic and other properties of the materials used in the device. Detailed numerical models have been developed for this purpose, e.g., ETFOS, http://www.ccp.zhwin.ch/etfos

${ }^{18}$ D. Berner et al., J. Mater. Chem. 16, 4468 (2006). 